\title{
Inibidores da bomba de prótons: Revisão e análise farmacoeconômica
}

\author{
Muriele Picoli Braga*, Cristiane de Bona da Silva**, Andréa Inês \\ Horn Adams***
}

RESUMO: Os inibidores da bomba de prótons (IBPs), são medicamentos que inibem a enzima $\mathrm{H}^{+}, \mathrm{K}^{+}$ATPase (ou bomba de prótons) realizando a supressão ácida gástrica. Atualmente, são comercializados seis representantes desta classe: omeprazol, lansoprazol, pantoprazol, rabeprazol, esomeprazol e dexlansoprazol. Um novo IBP, tenatoprazol, está ainda em fase de desenvolvimento. 0 presente estudo propõe-se a revisar os estudos disponíveis a fim de expor informações sobre os IBPs e realizar um estudo farmacoeconômico entre doses-padrão desses medicamentos para o tratamento da úlcera péptica gastroduodenal. Evidências atuais sugerem que os IBPs possuem eficácia semelhante no tratamento de várias desordens gástricas e no protocolo de erradicação do Helicobacter pylori. A partir da análise farmacoeconômica ora realizada, constatou-se que o lansoprazol apresenta perfil mais favorável.

Descritores: Inibidores da bomba de prótons, Estudo farmacoeconômico.

\section{Proton Pump Inhibitors: Review and Analysis Pharmacoeconomic}

ABSTRACT: The proton pump inhibitors (PPIs) are drugs that inhibit the enzyme $\mathrm{H}+, \mathrm{K}+-\mathrm{ATPase}$ (or proton pump), by performing gastric acid suppression. Currently, there are five marketed drugs of this class: omeprazole, lansoprazole, pantoprazole, rabeprazole and esomeprazole. A new PPI, tenatoprazole, is still under development. This study aims to review the available studies in order to reveal information about the PPIs and to perform a pharmacoeconomic study between standard doses of these drugs, employed in the treatment of gastroduodenal peptic ulcer. Current evidence suggests that PPIs have similar efficacy in the treatment of various gastric disorders and in the eradication of Helicobacter pylori. Through the pharmacoeconomic analysis done, it was found that lansoprazole presents a more favorable profile.

Descriptors: Proton pump inhibitors; Pharmacoeconomic study.

\footnotetext{
*Mestranda no Programa de pós-graduação em Ciências Farmacêuticas pela Universidade Federal de Santa Maria (UFSM), Santa Maria, RS, Brasil.

**Pós-doutora em Ciências da Saúde (Nanotecnologia) pela Universidade Federal do Rio Grande do Sul (UFRGS), Rio Grande, RS, Brasil e professora adjunta no Departamento de Farmácia Industrial da Universidade Federal de Santa Maria (UFSM), Santa Maria, RS, Brasil.

***Doutora em Ciências Farmacêuticas pela Universidade Federal do Rio Grande do Sul (UFRGS), Rio Grande, RS, Brasil e professora adjunta no Departamento de Farmácia Industrial da Universidade Federal de Santa Maria (UFSM), Santa Maria, RS, Brasil.
} 


\section{Introdução}

$\mathrm{O}$ reconhecimento de que a enzima $\mathrm{H}^{+}, \mathrm{K}^{+}$- Adenosina Tri-Fosfatase $\left(\mathrm{H}^{+}, \mathrm{K}^{+}\right.$-ATPase ou bomba de prótons) constitui a etapa final da secreção ácida levou ao desenvolvimento de uma classe de fármacos, a dos inibidores da bomba de prótons (IBPs), usados no tratamento de distúrbios gastrintestinais. ${ }^{1}$

Atualmente, existem sete representantes desta classe, dos quais seis são comercializados (Figura 1): omeprazol, lansoprazol, pantoprazol, rabeprazol, esomeprazol (isômero $S$ do omeprazol), dexlansoprazol (enantiômero do lansoprazol, ainda não comercializado no Brasil) e o tenatoprazol..$^{2,3}$

Figura 1 - Estrutura química dos inibidores da bomba de prótons

$\perp$
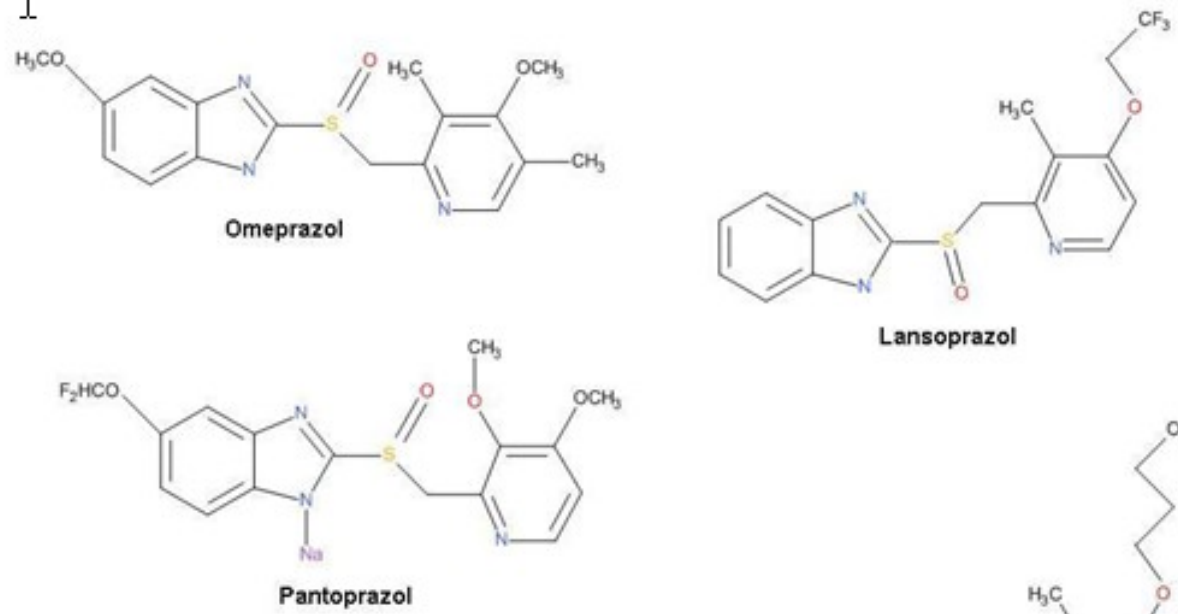

Lansoprazol
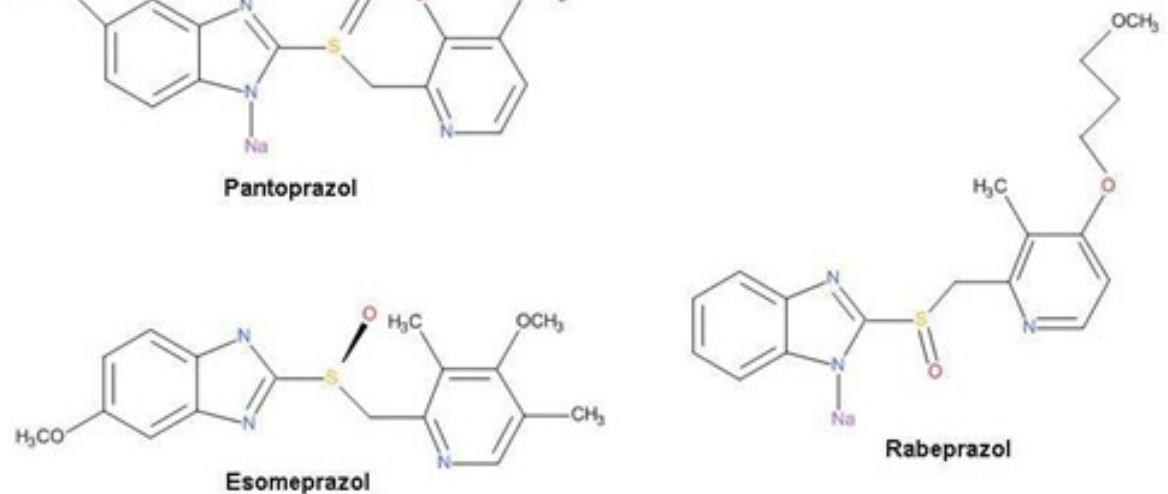

Esomeprazol

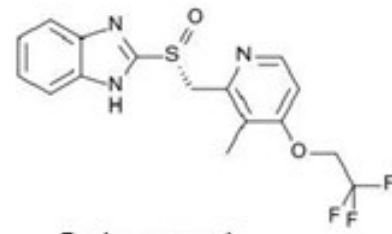

Dexlansoprazol

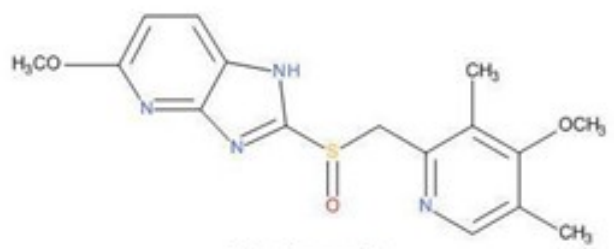

Tenatoprazol
Saúde (Santa Maria), Ahead of Print, v.37, n.2, p. 19-32, 2011. Inibidores da bomba de prótons: Revisão e análise farmacoeconômica ISSN 2236-5834 imidazopiridínico no lugar do anel benzimidazólico, o que aumenta seu tempo de permanência no organismo, sendo importante, principalmente, para o controle da acidez durante a noite. ${ }^{4}$ Porém, ainda se desconhece a importância clínica dessa característica farmacocinética. ${ }^{5}$ Todos compartilham da mesma estrutura, um anel a-piridilmetilsulfinil 
benzimidazol substituído, mas variam em termos de substituições nos grupos piridina ou benzimidazol. Eles também têm em comum os mecanismos inibitórios e são todos bases fracas protonáveis em $\mathrm{pK}_{a}$ de cerca de 4 a 5 , o que faz com que eles se acumulem seletivamente no espaço ácido das células parietais secretoras. ${ }^{1}$

Muitos fármacos diferentes, como os antagonistas dos receptores $\mathrm{H}_{2}$, também inibem a secreção ácida. Mas os inibidores da bomba de prótons têm sido considerados mais eficazes, pois bloqueiam a etapa final da secreção ácida. ${ }^{6-8}$ Atualmente, os inibidores da bomba de prótons são a segunda classe de medicamentos mais comumente prescritos nos Estados Unidos. $^{2}$

Esse trabalho propõe-se a revisar estudos disponíveis na literatura sobre os IBPs bem como, realizar um estudo farmacoeconômico entre doses-padrão utilizadas no tratamento da úlcera péptica gastroduodenal, por ser uma indicação comum dos mesmos.

\section{Fisiopatologia da úlcera péptica}

A úlcera péptica é uma das principais indicações clínicas para reduzir a secreção de suco gástrico.9 Uma úlcera péptica é uma ferida bem definida, circular ou oval, formada no revestimento do trato gastrintestinal. Os nomes das úlceras identificam a sua localização anatômica ou as circunstâncias em que se desenvolvem. A úlcera duodenal, o tipo mais comum de úlcera péptica, surge no duodeno. As úlceras gástricas normalmente situam-se na parte alta da curvatura do estômago. ${ }^{10}$

A fisiopatologia da úlcera péptica pode ser considerada um desequilíbrio entre os fatores de defesa da mucosa (bicarbonato, mucina, prostaglandina, óxido nítrico, outros peptídios e fatores de crescimento) e fatores agressivos (ácido e pepsina)..$^{11} \mathrm{~A}$ regulação da secreção de ácido pelas células parietais é especialmente importante na patogênese da úlcera péptica e constitui um alvo para a ação de fármacos. A secreção das células parietais é uma solução isotônica de $\mathrm{HCl}(150 \mathrm{mmol} / \mathrm{L}) \mathrm{com} \mathrm{pH}$ abaixo de 1. De modo bastante simplificado, íons cloreto $\left(\mathrm{Cl}^{-}\right)$são transportados ativamente do plasma para a célula parietal. Esta secreção de íons $\mathrm{Cl}$ - é acompanhada por íons $\mathrm{K}+$, que são, então, trocados por íons $\mathrm{H}^{+}$pela $\mathrm{K}^{+}-\mathrm{H}^{+}-$ ATPase. Tem-se então a combinação de íons hidrogênio $\left(\mathrm{H}^{+}\right)$e cloreto $(\mathrm{Cl})$, seguido de sua liberação pelas glândulas secretoras gástricas. ${ }^{9}$

O desenvolvimento de úlceras pépticas está relacionado à infecção da mucosa do estômago por Helicobacter pylori (bacilo gram-negativo que causa gastrite crônica), bem como ao uso de certos medicamentos (sobretudo o ácido acetilsalicílico, o ibuprofeno e outros antiinflamatórios não-esteróides), os quais provocam erosões e úlceras no estômago, especialmente nas pessoas de idade avançada. Esses efeitos, no entanto, são revertidos pela interrupção do tratamento, sendo a recidiva pouco provável, a menos que se reinicie 0 mesmo tratamento. ${ }^{10}$ As prostaglandinas (principalmente $\mathrm{E}_{2}$ e $\mathrm{I}_{2}$ ), sintetizadas na mucosa gástrica principalmente pela enzima ciclooxigenase-1 (COX-1), estimulam a secreção de muco e de bicarbonato, diminuem a secreção de ácido e causam vasodilatação. Todas essas ações servem para proteger o estômago contra lesões. Isto, provavelmente, explica a capacidade de muitos antiinflamatórios não-esteróides (AINEs) inespecíficos de causar sangramento e erosões gástricas, visto que são inibidores da COX-1. ${ }^{9}$

Saúde (Santa Maria), Ahead of Print v.37, n.2, p. 19-32, 2011. Braga, M. P., Silva, C. B., Adams, A. I. H.

A terapia da úlcera péptica alia a redução da secreção de ácido gástrico usando 
antagonistas dos receptores $\mathrm{H}_{2}$ (cimetidina, ranitidina, nizatidina e famotidina) ou IBPs, bem como a neutralização do ácido secretado com antiácidos (hidróxido de magnésio, hidróxido de alumínio e bicarbonato de sódio) e protetores da mucosa, como o sucralfato. Embora os antiácidos tenham sido utilizados ao longo do tempo e tenham provado ser relativamente eficazes, o uso dos mesmos é inconveniente devido à necessidade de várias doses diárias e também pela associação a efeitos colaterais indesejados. ${ }^{10}$ No caso de infecção por $H$. pylori, são associados anti-secretores e antibióticos (claritromicina, amoxicilina, metronidazol, tinidazol, tetraciclina e fluorquinolonas, como levofloxaxino e moxifloxacino). 0 tratamento conhecido como terapia tríplice (combinação de um IBP, claritromicina e amoxicilina) começou a apresentar evidências de resistência microbiana por volta do ano 2000, com taxas de cura inferiores a $80 \%$. Atualmente, os estudos apontam para a eficácia de terapia concomitante (associação de amoxicilina, claritromicina, tinidazol ou metronidazol e IBP, por 10-14 dias), terapia seqüencial (amoxicilina + IBP/5 dias, seguida de claritromicina e tinidazol ou metronidazol + IBP/5 dias), terapia concomitante-combinada (combinação das duas citadas anteriormente) ou ainda, terapia com bismuto (associação de bismuto, tetraciclina, metronidazol + IBP). A terapia tríplice ainda permanece como alternativa quando há evidências de susceptibilidade à claritromicina. ${ }^{12}$

\section{Inibidores da bomba de prótons}

\section{Usos}

Em 1989 foi introduzido na terapêutica o primeiro inibidor da bomba de prótons, o omeprazol. Após surgiram outros fármacos, com propriedades físico-químicas e farmacológicas semelhantes: pantoprazol, rabeprazol, esomeprazol e mais recentemente 0 dexlansoprazol. Os fármacos desta classe são considerados muito eficazes e relativamente equivalentes durante o tratamento, reduzindo em até $95 \%$ a produção diária de ácido gástrico. ${ }^{13}$

Os IBPs produzem uma supressão ácida significativamente mais eficaz e prolongada do que os antagonistas dos receptores $\mathrm{H}_{2}$ e são capazes de manter o pH intragástrico superior a 4 por até 16 a $18 \mathrm{~h} / \mathrm{dia}$. Foi demonstrado que a cura de doenças relacionadas com o excesso de ácido está diretamente relacionada com o grau e a duração da supressão ácida e com a duração do tratamento. ${ }^{14,15}$ Dessa forma, seu uso é recomendado em diretrizes atuais para 0 tratamento da doença do refluxo gastroesofágico ${ }^{13}$, para a prevenção de úlcera péptica em pacientes recebendo agentes inflamatórios não esteróides (AINEs), em protocolos de erradicação da Helicobacter pylori, ${ }^{5}$ na síndrome de Zollinger-Ellison, gastrinomas, esofagite e gastrite. ${ }^{16}$

A Tabela 1 expõe de maneira sucinta evidências contemporâneas sobre a eficácia do tratamento com inibidores da bomba de prótons. ${ }^{5}$

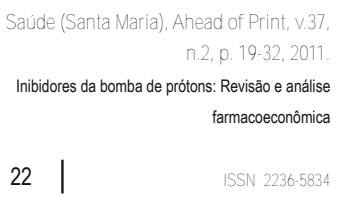




\section{Eficácia comprovada}

Tratamento sintomático e cicatrização de úlceras pépticas induzidas por uso crônico de AINEs.

Prevenção de lesões gastrintestinais induzidas pelo uso crônico de AINEs.

Coadjuvante na prevenção de recorrência de úlceras pépticas induzidas por H. pylori.

Tratamento de esofagite ou sintomas associados à doença do refluxo gastresofágico.

\begin{tabular}{c} 
Provável eficácia \\
Tratamento da dispepsia com pirose e regurgitação. \\
Sugerida ineficácia \\
Tratamento do sangramento digestivo alto causado por úlcera péptica. \\
Profilaxia primária de sangramento digestivo alto causado por úlcera de estresse. \\
\hline
\end{tabular}

Além da atividade anti-secretora, os IBPs demonstram uma importante atividade antiHelicobacter pylori in vitro, tendo um efeito sinérgico com antibióticos. ${ }^{18} 0$ mecanismo para 0 efeito sinérgico ainda não é totalmente claro, mas pode ser devido à inibição da enzima urease bacteriana, um importante fator de proteção contra o meio ácido, ou devido à ligação à enzima $\mathrm{H}^{+}, \mathrm{K}^{+}$-ATPase da bactéria, a qual auxilia na manutenção do gradiente transmembrana. ${ }^{19,20}$

\section{Farmacocinética}

Todos os inibidores da bomba de prótons são "pró-fármacos" que necessitam ativação em ambiente ácido. Os fármacos dessa classe entram na célula parietal a partir do sangue e acumulam-se nos canalículos secretores ácidos da célula parietal, onde são ativados por um processo catalisado por prótons, que resulta na formação de uma sulfenamida tiofilica ou ácido sulfênico. Essa forma ativada reage por meio de ligação covalente com o grupo sulfidril de cisteínas do domínio extracelular da $\mathrm{H}^{+}, \mathrm{K}^{+}$-ATPase, as quais são essenciais para a inibição da produção ácida. ${ }^{11}$

0 resultado é a inibição irreversível da secreção ácida da bomba de prótons. ${ }^{21}$ Por causa da ligação covalente, os efeitos inibitórios dos IBPs duram muito mais tempo do que sua meia-vida plasmática. ${ }^{2}$ A meia-vida plasmática dos IBPs varia em torno de 1 a 2 horas, e o efeito sobre a secreção ácida pode durar até 24 horas, até que ocorra a síntese de nova enzima. Todo o potencial inibidor do ácido pode levar até 3 a 4 dias. ${ }^{21}$ Por exemplo, a restauração da biossíntese da enzima $\mathrm{H}^{+}, \mathrm{K}^{+}$-ATPase ocorre cerca de 96 horas após a dose oral de pantoprazol. ${ }^{22}$

De acordo com o mecanismo de ação, os anéis piridina e benzimidazol, bem como a cadeia $-\mathrm{CH}_{2}$-SO- são essenciais para a atividade dos IBPs. Os substituintes em ambos os anéis são importantes para determinar a velocidade de conversão à forma ativa e diminuir a toxicidade. ${ }^{23,24}$

São rapidamente absorvidos, altamente ligados às proteínas e geralmente bem tolerados, mas de modo semelhante a alguns bloqueadores $\mathrm{H}_{2}$, interagem com algumas enzimas microssomais do citocromo P450, podendo reduzir o catabolismo alguns fármacos, como varfarina e fenitoína. 25,11 Uma vez que causam inibição profunda e duradoura da saúde (Santa Maria), Ahead of Print v.37, secreção ácida gástrica, teoricamente é possível que possam interferir na absorção de fármacos em que o pH gástrico seja um fator importante para a biodisponibilidade (p. ex.: cetoconazol, ésteres da ampicilina, sais de ferro, digoxina). ${ }^{26}$ 
Apesar de pertencerem à mesma classe terapêutica, existem algumas diferenças em seus perfis farmacológicos. Ao contrário do omeprazol e do lansoprazol, o pantoprazol tem um perfil farmacocinético linear (e, portanto mais previsível), biodisponibilidade menos variável e um rápido início de ação. ${ }^{27}$

Por ser uma base mais forte, o rabeprazol converte-se mais rapidamente à forma sulfenamida, resultando em uma rápida inibição da enzima. Isto se deve ao valor de pKa do nitrogênio do seu anel piridínico ( $\mathrm{pKa}=5$ ), enquanto que $\mathrm{o}$ dos demais apresentam valor em torno de 4. . $^{19,28}$

Outra diferença é em relação à atividade anti-Helicobacter pylori, onde o rabeprazol mostrou ser 10 vezes mais potente que omeprazol e lansoprazol na inibição da enzima urease bacteriana. Este efeito provavelmente é devido à formação de pontes dissulfeto no sítio ativo da enzima. ${ }^{29}$ Além disso, rabeprazol e seu metabólito tioéter possuem marcada atividade inibitória sobre a motilidade de $H$. pylori, a qual é importante para colonização da mucosa gástrica. ${ }^{30}$

Seguindo nas diferenças farmacocinéticas dos IBPs, tem-se o esomeprazol (isômero $S$ do omeprazol), considerado o primeiro inibidor de bomba de prótons desenvolvido como um único isômero para 0 tratamento de doenças ácido-relacionadas. ${ }^{31} \mathrm{~A}$ justificativa para 0 desenvolvimento de isômeros únicos dos IBPs decorre do fato de que a quiralidade de fármacos pode ter um significado clínico considerável, uma vez que as enzimas e receptores geralmente têm uma preferência estereoquímica de um isômero óptico. Isso pode resultar em diferenças importantes no perfil farmacocinético e farmacodinâmico dos isômeros ópticos, e, portanto, na sua eficácia clínica. ${ }^{32} \mathrm{O}$ esomeprazol possui uma depuração mais lenta comparado com a forma racêmica e, portanto, o esperado é que se atinja um maior nível sanguíneo e melhor efeito inibitório da bomba de prótons. Em dois estudos que compararam os efeitos do pH intragástrico do esomeprazol com omeprazol, foram encontradas pequenas diferenças, mas estatisticamente significativas entre os dois. ${ }^{33,34}$

O dexlansoprazol foi aprovado em 2009 pelo FDA e ainda não é comercializado no Brasil, sendo o primeiro inibidor da bomba de prótons com uma formulação de liberação lenta que contém dois tipos de grânulos com revestimento entérico, resultando em picos de concentração em 1 a 2 horas após a administração e novamente em 4 a 5 horas. ${ }^{35}$

Estudos farmacocinéticos mostraram que o tenatoprazol exibe um longo tempo de meiavida (9 horas para a administração repetida de $40 \mathrm{mg}$, isto é, de 5 a 7 vezes maior que os outros IBPs disponíveis atualmente) e investigações farmacodinâmicas têm mostrado um aumento do $\mathrm{pH}$ intragástrico com tenatoprazol $(\mathrm{pH} \mathrm{4,6)}$ significativamente mais elevado do que o observado com o mesmo regime de esomeprazol ( $\mathrm{pH} \mathrm{4,2).} \mathrm{Evidenciou-se} \mathrm{também} \mathrm{que}$ o efeito inibitório da supressão ácida com tenatoprazol é mais duradouro, o que foi atribuído à estrutura química diferente de tenatoprazol e/ou às características resultantes da interação com o sítio da cisteína ou sítios da $\mathrm{H}^{+}, \mathrm{K}^{+}$-ATPase. ${ }^{28,36}$

Apesar de diferenças e/ou vantagens farmacocinéticas, todos os IBPs inibem fortemente a secreção de ácido gástrico, não demonstrando diferenças terapêuticas significativas entre si. $^{5}$

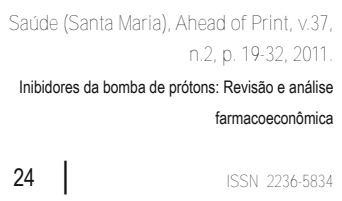




\section{Formas farmacêuticas e estabilidade}

Todos os inibidores da bomba de prótons são instáveis quando expostos a meio ácido, como o meio do estômago. Portanto, eles são formulados com revestimento entérico que protege o fármaco do ambiente ácido gástrico. ${ }^{37,38} \mathrm{As}$ formas farmacêuticas disponíveis no mercado são: injetáveis, comprimidos com revestimento entérico de liberação prolongada, comprimidos de desintegração rápida, cápsulas com grânulos com revestimento entérico de liberação normal e prolongada.

Os comprimidos com revestimento entérico ou grânulos com cobertura entérica (pellets gastrorresistentes), encapsulados em uma camada externa de gelatina, passam pelo ambiente ácido do estômago intactos. 0 revestimento entérico dissolve-se quando entra no ambiente alcalino do intestino delgado, onde o fármaco é absorvido, sendo assim evitada a degradação dos fármacos pelo ácido do esôfago e estômago. ${ }^{21}$

Desse modo, deve-se ter cuidado na administração oral quando se torna necessário uma preparação líquida, como no caso de crianças ou de pacientes hospitalizados. Muitas vezes é realizada uma preparação extemporânea a partir do conteúdo de uma cápsula (os pellets) solubilizado em solvente adequado. Porém, ao esmagar os pellets, a camada protetora é destruída, expondo o fármaco para o $\mathrm{pH}$ gástrico e, dessa forma, promovendo sua degradação. Do mesmo modo, uma formulação intravenosa não é ativa quando administrada por via oral. ${ }^{39}$

Avaliou-se a estabilidade de omeprazol oral líquido $(2 \mathrm{mg} / \mathrm{mL})$ preparado a partir do conteúdo de cápsulas de omeprazol, o qual foi dissolvido em bicarbonato de sódio para injeção $8,4 \%$. As amostras preparadas permaneceram estáveis por 30 dias a $-20^{\circ} \mathrm{C}$ e $4^{\circ} \mathrm{C}$, apresentando menos de $3 \%$ e $5 \%$ de perda, respectivamente. Além disso, não houve alteração no aspecto do líquido armazenado nestas temperaturas. No entanto, as amostras armazenadas à temperatura ambiente apresentaram perda de $8 \%$ em 14 dias e perda de 14\% em 18 dias e a cor da preparação mudou de branco para marrom durante 0 armazenamento. ${ }^{39}$

Recentemente foi introduzido na clínica comprimido de desintegração oral, uma formulação alternativa de lansoprazol. É fácil de engolir e pode ser tomado com ou sem água. Este recurso melhora o cumprimento da terapia quando os pacientes estão fora de casa (e quando a água não está prontamente disponível), em doentes com disfagia ou odinofagia, bem como distúrbios de deglutição. A farmacocinética de lansoprazol oral após a administração do comprimido é essencialmente idêntica à observada após a dose com cápsulas de grânulos. ${ }^{40}$

\section{Doses e Posologia}

Doses-padrão de IBPs podem reduzir a secreção de ácido gástrico em até $98 \%$ por inativação irreversível da bomba de prótons da célula parietal gástrica. Recomenda-se que sejam administrados aproximadamente 1 hora antes das refeições, para aumentar a biodisponibilidade. ${ }^{21} \mathrm{~A}$ alimentação reduz 0 pico de concentração do lansoprazol e sua absorção em aproximadamente $50 \%{ }^{26}$ No entanto, a ingestão concomitante de alimento não influi significativamente na biodisponibilidade do pantoprazol em dose simples em pessoas saudáveis. ${ }^{41}$

Saúde (Santa Maria), Ahead of Print v.37, n.2, p. 19-32, 2011.

Braga, M. P., Silva, C. B., Adams, A. I. H. 
Estudos envolvendo omeprazol (20mg), pantoprazol (40 mg), lansoprazol (30 mg), rabeprazol $(20 \mathrm{mg})$ e esomeprazol $(20 \mathrm{mg})$ apontam a equivalência clínica destes fármacos nestas dosagens. ${ }^{2}$ Para 0 tratamento da maior parte dos distúrbios relacionados, esses fármacos são administrados em única dose diária.

\section{Efeitos adversos}

Os IBPs são geralmente bem tolerados pelos pacientes. Dor de cabeça, dor abdominal, náusea e diarréia são os efeitos colaterais mais comuns. A incidência de diarréia aumenta com a idade e dosagem. Efeitos secundários pouco freqüentes incluem erupção cutânea, coceira e constipação. A incidência global de efeitos secundários é inferior a $5 \%$, fazendo 0 uso de IBPs ideal para a terapia de curto prazo; no entanto, continua a existir preocupações sobre 0 impacto a longo prazo. 0 efeito de primeira passagem e a depuração renal são mínimos, portanto, a redução da dose não é necessária para pacientes com insuficiência renal. ${ }^{42}$

Na dose habitual (20 a $40 \mathrm{mg} / \mathrm{dia}$ ) o omeprazol inibe acima de $90 \%$ da secreção ácida de 24 horas na maioria dos pacientes, tornando muitos destes pacientes quase aclorídricos. Devido a esta potência, há dúvidas na segurança do seu uso em longo prazo. 0 ácido gástrico, por um mecanismo de "feedback", inibe a secreção endócrina da gastrina pelas células $\mathrm{G}$ localizadas nas glândulas antrais (pilóricas). Se a acidez gástrica é nitidamente reduzida, as células $G$ secretam quantidades aumentadas de gastrina, levando à hipergastrinemia. A gastrina é um hormônio trófico, estimula a proliferação e crescimento de certas células e tecidos. A utilização em longo prazo de omeprazol em humanos pode possivelmente produzir proliferação de células e tumores carcinóides, porém não foram ainda claramente estabelecidos como uma conseqüência do uso crônico do medicamento. ${ }^{25}$

A administração de pantoprazol em longo prazo (24 meses) em animais (ratos e camundongos) causou a inibição da secreção ácida gástrica, levando a hipergastrinemia, resultando na hiperplasia das células e observando-se tumores carcinóides do estômago e cólon nos animais testados. Estes fenômenos não foram constatados em seres humanos. ${ }^{43}$

Embora a segurança do uso dos IBPs já tenha sido comprovada, relatos recentes têm chamado a atenção para potenciais efeitos secundários ou interações medicamentosas. Estudos apontam para absorção deficiente de alguns fármacos quando administrados juntamente com os IBPs, tais como: cetoconazol, itraconazol e astranavir, bem como aumento na absorção de medicamentos como nifedipino e digoxina. ${ }^{13}$

Além disso, estudos recentes levantaram a questão de que, quando usados em terapia conjunta, os IBPs levam a uma redução na conversão de clopidogrel em sua forma ativa, reduzindo assim a eficácia do tratamento com clopidogrel, que é um importante agente antiagregante plaquetário. Dessa forma, os IBPs estariam contribuindo para a ineficácia do tratamento de cardiopatias e eventos aterotrombóticos. ${ }^{13,44}$ Por outro lado, autores citam que os estudos existentes apresentam resultados inconsistentes e discordantes. Mesmo assim, em novembro de 2009, o FDA emitiu um alerta aos profissionais de saúde sobre o potencial

Saúde (Santa Maria), Ahead of Print, v.37, n.2, p. 19-32, 2011. farmacoeconômica uso desses dois IBPs. ${ }^{4}$

Recentemente, também foi levantada a questão de que o uso de IBPs poderia induzir má absorção de cálcio pelos ossos e, dessa forma, levar à osteoporose e aumentar o risco da 
ocorrência de fraturas, principalmente no quadril. No entanto, os dados apresentados não são uniformes. Alguns estudos conseguem demostrar que há uma pequena, mas estatísticamente significativa, associação entre o uso de IBPs e o risco de fraturas, porém não demonstram significância clínica pelo fato do risco ser baixo. Mesmo com as limitações metodológicas dos estudos, o FDA anunciou em maio de 2010, que deveria existir uma mudança na bula dos IBPs, contendo a informação de indicar um possível risco de fratura com o uso desses medicamentos. ${ }^{44}$

\section{Análise farmacoeconômica dos inibidores da bomba de prótons}

Farmacoeconomia é um termo relativamente recente que se refere à aplicação da economia ao estudo dos medicamentos. Surgiu em países desenvolvidos no final dos anos 80 e seu corpo de conhecimento encontra-se fundamentado na economia da saúde, que visa melhorar a eficiência dos gastos nos sistemas de saúde. ${ }^{45}$

Os estudos farmacoeconômicos abrangem a identificação, o cálculo e a comparação dos custos (recursos consumidos), riscos e desfechos (clínicos, econômicos e humanísticos) de programas, serviços ou terapias e a determinação de alternativas que produzam os melhores resultados diante dos recursos utilizados. ${ }^{46}$ Esses estudos podem também ser utilizados para avaliar procedimentos, produtos médico-hospitalares e outros itens utilizados com fins terapêuticos. ${ }^{47}$

Autores clássicos categorizam quatro tipos de análises farmacoeconômicas, que têm em comum o elemento custo e diferem quanto à unidade de desfecho, sendo elas: análise de minimização de custo (AMC), análise de custo-efetividade (ACE), análise de custoutilidade (ACU) e análise de custo-benefício (ACB). ${ }^{46,48}$ Tendo em vista que o objetivo deste estudo é avaliar os custos de um certo tratamento com a aplicação dos diferentes IBPs disponíveis no mercado, foi utilizada a análise de minimização de custo.

A AMC é o tipo de análise farmacoeconômica mais simples, que utiliza como meio de comparação única e exclusivamente o custo, tendo em vista que as opções terapêuticas são igualmente efetivas. Esta abordagem é justificada quando as alternativas de programas ou terapias comparadas produzem resultados clínicos equivalentes, como em tomadas de decisões de guias terapêuticos. ${ }^{49,50}$ Assim, o primeiro passo crítico antes de conduzir a AMC é determinar a equivalência terapêutica das intervenções. ${ }^{50}$

Dessa forma, analisou-se a posologia habitualmente recomendada para obtenção da cicatrização da úlcera péptica gastroduodenal. Foram utilizados os custos relativos aos medicamentos de referência, pois rabeprazol e esomeprazol ainda não possuem o seu medicamento genérico correspondente, e, ainda, foram selecionadas as embalagens que continham o maior número de formas farmacêuticas disponíveis no mercado, visando uma maior redução dos custos e padronização da análise. Analisaram-se somente os dados relativos aos medicamentos comercializados no Brasil.

Para comparar o custo da terapia é necessário considerar a quantidade de cápsulas ou comprimidos utilizados por dia, a duração média da terapia, e a aquisição desse material. ${ }^{49}$

A Tabela 2 ilustra a comparação das opções terapêuticas. Os dados relacionados às estratégias terapêuticas foram retirados das bulas dos medicamentos de referência.

Saúde (Santa Maria), Ahead of Print v.37, n.2, p. 19-32, 2011 Braga, M. P., Silva, C. B., Adams, A. I. H. 
Tabela 2 - Análise de minimização de custo dos IBPS.

\begin{tabular}{|c|c|c|c|c|c|c|}
\hline & $\begin{array}{c}\text { Preço } \\
\text { da } \\
\text { embalagem* }^{*}\end{array}$ & $\begin{array}{c}\text { Unidades } \\
\text { por } \\
\text { embalagem }\end{array}$ & $\begin{array}{l}\text { Preço } \\
\text { unitário }\end{array}$ & $\begin{array}{l}\text { Tempo de } \\
\text { tratamento }\end{array}$ & $\begin{array}{c}\text { Custo do tratamentof } \\
\text { (se houver } \\
\text { fracionamento) }\end{array}$ & $\begin{array}{r}\text { Custo do } \\
\text { tratamento } \\
\text { (embalagem } \\
\text { fechada) }\end{array}$ \\
\hline $\begin{array}{l}\text { Omeprazol } \\
(20 \mathrm{mg})\end{array}$ & $\mathrm{R} \$ 57,54$ & 28 cáps. & $\mathrm{R} \$ 2,05$ & 6 semanas $\dagger$ & $\mathrm{R} \$ 86,10$ & $\mathrm{R} \$ 115,08$ \\
\hline $\begin{array}{l}\text { Lansoprazol } \\
\qquad(30 \mathrm{mg})\end{array}$ & $\mathrm{R} \$ 22,01$ & 14 cáps. & $\mathrm{R} \$ 1,57$ & 6 semanas $\dagger$ & $\mathrm{R} \$ 66,04$ & $\mathrm{R} \$ 66,04$ \\
\hline $\begin{array}{l}\text { Pantoprazol } \\
\qquad(40 \mathrm{mg})\end{array}$ & $\mathrm{R} \$ 182,55$ & 28 comp. & $\mathrm{R} \$ 6,52$ & 4 semanas & $\mathrm{R} \$ 182,56$ & $\mathrm{R} \$ 182,56$ \\
\hline $\begin{array}{l}\text { Rabeprazol } \\
\qquad(20 \mathrm{mg})\end{array}$ & $\mathrm{R} \$ 183,68$ & 28 comp. & $\mathrm{R} \$ 6,56$ & 6 semanas & $R \$ 275,52$ & $\mathrm{R} \$ 367,36$ \\
\hline $\begin{array}{l}\text { Esomeprazol } \\
\qquad(20 \mathrm{mg})\end{array}$ & $\mathrm{R} \$ 148,01$ & 28 comp. & $\mathrm{R} \$ 5,28$ & 6 semanas $\dagger$ & $\mathrm{R} \$ 221,76$ & $\mathrm{R} \$ 296,02$ \\
\hline
\end{tabular}

* Preços referentes à pesquisa de mercado realizada em farmácias locais † média do tempo total de tratamento ( 4 a 8 semanas)

‡ estimado de acordo com a posologia diária recomendada (1 comp. ou 1 cáp. por dia, para todos os IBPs)

A análise da Tabela 2 aponta que o preço unitário e o custo diário da terapia são mais caros com a utilização do rabeprazol e, além disso, o custo do tratamento por embalagem é cerca de 6 vezes mais caro com o emprego do rabeprazol comparado ao tratamento de menor custo, o lansoprazol. Ainda, neste caso, o paciente necessita comprar duas embalagens fechadas para a realização do tratamento por completo e há sobra de comprimidos, o que tornaria a terapia com lansoprazol novamente mais vantajosa onde, ao adquirir três embalagens fechadas para o cumprimento da terapia, o paciente conta com 0 número exato de cápsulas utilizadas em todo o tratamento, sem sobra de medicamento e sem gastos desnecessários.

\section{Considerações finais}

Os inibidores da bomba de prótons consistem em medicamentos usados muitas vezes empiricamente (por prescrição ou automedicação) para tratamento das manifestações digestivas que lembram as das doenças já referidas ou na prevenção do surgimento de tais sintomas. São considerados o maior avanço no tratamento de doenças gástricas. Quando usados corretamente, os IBPs são sem dúvida os mais potentes inibidores da secreção ácida gástrica disponíveis e, portanto, tornaram-se essenciais na terapia de várias doenças ácidorelacionadas.

Saúde (Santa Maria), Ahead of Print, v.37, n.2, p. 19-32, 2011. Iibidores da bomba de prótons: Revisão e análise farmacoeconômica

Evidências atuais sugerem que os IBPs possuem eficácia semelhante. Estudos realizados com o tenatoprazol (fase pré-clínica) sugerem maior eficácia no controle da supressão da secreção gástrica noturna. Porém, estudos clínicos ainda são necessários para confirmar esta hipótese e torná-lo disponível para uso. 
Com base na análise farmacoeconômica realizada, comparando os custos dos IBPs disponíveis aplicados no tratamento da úlcera péptica gastroduodenal, o lansoprazol fornece um perfil mais favorável de farmacoeconomia. Portanto, a substituição de fármacos mais antigos por representantes mais novos acarreta, no mínimo, em custo desnecessário.

O levantamento bibliográfico realizado apontou a baixa incidência de efeitos adversos do tratamento a curto prazo; no entanto, a segurança e ausência de toxicidade a longo prazo ainda não está devidamente documentada. Sendo assim, o uso indiscriminado dessa classe de fármacos deve ser evitado.

\section{Referências}

1. Wolfe MM. Pharmacological principles governing the use of proton pump inhibitors: tailoring therapy to improve GERD outcomes. Gastroenterol Clin. 2003:32:vii-ix.

2. James MM, Gabello M, Murray LJ, Farrell CP, Bellows J, Wolov KR et al. Proton pump inhibitors: actions and Reactions. Drug Discov Today. 2009:14:13-14.

3. Peura D. Dexlansoprazole: a new PPI formulation for treatment of GERD. Nat Rev Gastroenterol Hepatol. 2009:5(5):321-323.

4. Shin JM, Homerin M, Domagala F, Ficheux H, Sachs G. Characterization of the inhibitory activity of tenatoprazole on the gastric $\mathrm{H}+, \mathrm{K}+-\mathrm{ATP}$ ase in vitro and in vivo. Biochem Pharmacol. 2006:71:837-849.

5. Wannmacher, L. Inibidores da bomba de prótons: indicações racionais. Uso Racion. Medicam. 2004:2(1):16.

6. Lanzon-Miller S, Pounder RE, Hamilton RRE, Ball S, Chronos NAF, Raymond F, Olausson M, Cederberg C. Twenty-four hour intragastric acidity and plasma gastrin concentration before and during treatment with either ranitidine and omeprazole. Aliment Pharmacol Ther. 1987:1.

7. Burget DW, Stephen G, Chiverton SG, Hunt RH. Is there an optimal degree of acid suppression for healing of duodenal ulcer? A model of the relationship between ulcer healing and acid suppression. Gastroenterol. 1990:99:345-351.

8. Bell NJ, Burget D, Howden CW, Hunt RH. Appropriate acid suppression for the management of gastrooesophageal reflux disease. Digestion. 1993: 51(1):59-67.

9. Rang HP, Dale MM, Ritter JM. Farmacologia. 6ª ed. Rio de Janeiro: Elsevier, 2007.

10. Marmo MC, Neto UF. Úlcera Péptica Gastroduodenal. J Ped Gastroenterol, Nutrition Liver Diseases. 2005:9:1-13.

11. Hoogerwerf WA, Pasricha PJ. Agentes usados para o controle da acidez gástrica e no tratamento de úlceras pépticas e da doença do refluxo gastresofágico. In: Goodman, Gilman. As bases farmacológicas da terapêutica. 10ª ed. Rio de Janeiro: McGraw-Hill, 2005.

12. Rimbara E, Fischbach LA, Graham DY. Optimal therapy for Helicobacter pylori infections. Gastroenterol. Hepatol. 2011:8:79-88.

13. Varannes SB, Coron E, Galmiche JP. Short and long-term PPI treatment for GERD. Do we need morepotent anti-secretory drugs? Best Pract Res Cl Ga. 2010:24:905-921.

14. Jones DB, Howden CW, Burget DW et al. Acid suppression in duodenal ulcer: a meta-analysis to define optimal dosing with antisecretory drugs. Gut. 1987:28:1120-7.

Saúde (Santa Maria), Ahead of Print v.37, n.2, p. 19-32, 2011.

15. Bell NJ, Hunt RH. Role of gastric acid suppression in the treatment of gastrooesophageal reflux disease. Braga, M. P., Siva, C. B., Adams, A. A. . H. Gut. 1992:33: 118-24. 
16. Robinson M. Proton pump inhibitors: update on their role in acid-related gastrointestinal diseases. Int $\mathrm{J}$ Clin Pract. 2005:59:709-715.

17. Grube RR, May DB. Stress ulcer prophylaxis in hospitalized patients not in intensive care units. Am J Health Syst Pharm. 2007:64:1396-400.

18. Richardson P, Hawkey C, Stack W. Proton pump inhibitors - pharmacology and rationale for use in gastrointestinal disorders. Drugs. 1998:56(3):307-335.

19. Horn J. The proton pump inhibitors: similarities and differences. Clin Ther. 2000:22(3):266-280.

20. Galmiche JP et al. Tenatoprazole, a novel proton pump inhibitor with a prolonged plasma half-life: effects on intragastric $\mathrm{pH}$ and comparison with esomeprazole in healthy volunteers. Aliment Pharmacol Ther. 2004:19:655-662.

21. Katzung BG. Basic and clinical pharmacology. New York: Lange Medical Books/McGraw-Hill, 2004. p. $1037-8,1040-1$

22. Kromer W. Similarities and differences in the properties of substituted benzimidazoles: a comparison between pantoprazole and related compounds. Digestion. 1995:56:443-54.

23. Miner PBJR, Katz PO, Chen Y, Sostek M. Gastric acid control with esomeprazole, lansoprazole, omeprazole, pantoprazole, and rabeprazole: a five-way crossover study. Am J Gastroenterol. 2003:98:26162620.

24. Pedregal $C$, Avedaño $C$. Fármacos que alteran el transporte através de las menbranas celulares. In: Lopez MCA. Introduccion a la química farmacêutica. Madrid: Interamericana McGraw Hill, 1993. p. 360-362.

25. De Giacomo $\mathrm{C}$ et al. Omeprazole treatment of severe peptic disease associated with antal $\mathrm{G}$ cell hyperfunction and hyperpepsinogenemia I in an infant. J Pediatr. 1990:177:989.

26. Andersson T. Pharmacokinetics, metabolism and interactions of acid pump inhibitors. Focus on omeprazole, lansoprazole and pantoprazole. Clin Pharmacokinet. 1996:31:9-28.

27. Hartmann $M$, Theiss $U$, Huber $\mathrm{R}$ et al. Twenty-four-hour intragastric pH profiles and pharmacokinetics following single and repeated oral administration of the próton pump inhibitor pantoprazole in comparison to omeprazole. Aliment Pharmacol Ther. 1996:10:359-366.

28. Stedman CAM, Barclay ML. Review article: comparison of the pharmacokinetics, acid suppression and eficacy of proton pump inhibitors. Aliment Pharmacol Ther. 2000:14:963-978.

29. Barth J, Hahne W. Review article: rabeprazole-based therapy in Helicobacter pylori eradication. Aliment Pharmacol Ther. 2002:16(1):31-33.

30. Tsutsui N, Taneike I, Ohara T, Goshi et al. A novel action of the proton pump inhibitor rabeprazole and its thioether derivative against the motility of Helicobacter pylori. Antimicrob Agents Chemother. 2000: 44(11):3069-3073

31. Andersson $T$ et al. Pharmacokinetics and pharmacodynamics of esomeprazole, the S-isomer of omeprazole. Aliment Pharmacol Ther. 2001:15:1563-1569.

32. Creutzfeldt W. Chiral switch, a successful way for developing drugs: example of esomeprazole. Z Gastroenterol. 2000:38:893-7.

33. Lind T, Rydberg L, Kyleback A et al. Esomeprazole provides improved acid control vs. omeprazole in patients with symptoms of gastroesophageal reflux disease. Aliment Pharmacol Ther. 2000:14:861-867.

Saúde (Santa Maria), Ahead of Print, v.37, n.2, p. 19-32, 2011.

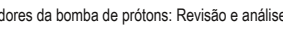

farmacoeconômica
34. Rohss $\mathrm{K}$, Hasselgren $\mathrm{G}$, Hedenstrom $\mathrm{H}$. Effect of esomeprazole $40 \mathrm{mg}$ vs omeprazole $40 \mathrm{mg}$ on 24-hour intragastric pH in patients with symptoms of gastroesophageal reflux disease. Dig Dis Sci. 2002:47:954-958.

35. Fass R, et al. The effect of dexlansoprazole MR on nocturnal heartburn and GERD-related sleep disturbances in patients with symptomatic GERD. Am J Gastroenterol. 2011:106:421-431. 
36. Hunt RH, Armstrong D, James C, Chowdhury SK, Yuan Y, Fiorentini P, Taccoen A, Cohen P. Effect on intragastric $\mathrm{pH}$ of a PPI with a prolonged plasma half-life: comparison between tenatoprazole and esomeprazole on the duration of acid suppression in healthy male volunteers. Am J Gastroenterol. 2005: 100:1949-1956.

37. Larson C, Cavuto NJ, Flockhart DA, Weinberg RB. Bioavailability and efficacy of omeprazole given orally and by nasogastric tube. Dig Dis Sci. 1996:41:475-479.

38. Chun AH, Erdman K, Zhang Y, Achari R, Cavanaugh JH. Effect on bioavailability of admixing the contents of lansoprazole capsules with selected soft foods. Clin Ther. 2000:22:231-236.

39. Trissel LA. Trissel's: Stability of Compounded Formulation. $2^{\mathrm{a}}$ ed. Washington: American Pharmaceutical Association, 2000.

40. Baldi F, Malfertheiner P. Lansoprazole fast disintegrating tablet: a new formulation for an established proton pump inhibitor. Digestion. 2003:67:1-5.

41. Huber R, Hartmann M, Bliesath $\mathrm{H}$ et al. Pharmacokinetics of pantoprazole in man. Int $\mathrm{J}$ Clin Pharmacol Ther. 1996:34:185-94.

42. Fitton A, Wiseman L. Pantoprazole. A review of its pharmacological properties and therapeutic use in acidrelated disorders. Drugs. 1996:51:460-82.

43. Vanderhoff MD, Tahboub RM. Proton pump inhibitors: an update. Clin Pharmacol. 2002:66:273-80.

44. Madanick RD. Proton pump inhibitor side effects and drug interactions: much ado about nothing? Clev Clin J Med. 2011:78(1):39-49.

45. Sacristán Del Castilho JA, Llach XB. Farmacoeconomia: evalución económica de medicamentos. Madrid: Ed. Medica; 1995.

46. Secoli SR. Farmacoeconomia da terapia analgésica utilizada na dor pós-operatória. São Paulo:[s.n.], 2002. Tese (Doutorado) - Universidade de São Paulo. Escola de Enfermagem, 2002.

47. Baskin LE. What is the difference between pharmacoeconomics and outcomes research? In: Baskin LE. Practical pharmaeconomics: how to design, perform and analyze outcomes research. Indianapolis (OH/USA): Advanstar Communications. 1998:1-4.

48. Drummond MF. The use of health economic information by reimbursement authorities. Rheumatol. 2003: 42(3):60-3.

49. Eisenberg JM. Clinical economics: a guide to economic analysis of clinical practices. JAMA. 1989:262(20):2879-86.

50. Robertson J, Lang D, Hill S. Use of pharmacoeconomics in prescribing research. Part 1: costs-moving beyond the acquisition price for drugs. J Clin Pharm Ther. 2003:28:73-79.

\section{Andréa Inês Horn Adams}

Endereço para correspondência - Departamento de Farmácia Industrial, Prédio 26, Centro de Ciências da Saúde - Campus UFSM.

Santa Maria, RS, Brasil - CEP: 97110-970

E-mail: andrea.ih.adams@gmail.com

Currículo lattes: http://lattes.cnpq.br/6872246935204149

Recebido em 30 de abril de 2011.

Aprovado em 26 de novembro de 2011.

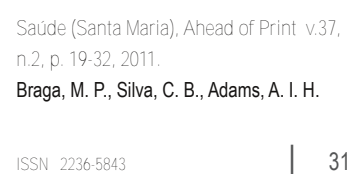


Saúde (Santa Maria), Ahead of Print, v.37,

n.2, p. 19-32, 2011.

Inibidores da bomba de prótons: Revisão e análise
$32 \mid$
ISSN 2236-5834 